\title{
Demanda de profesionales de educación superior en el área de Humanidades en la región 2 valles de Comayagua, Honduras (2015 - 2016)
}

\author{
Sandra Santos ${ }^{1}$ \\ Edwin Roldan Medina ${ }^{2}$
}

\section{RESUMEN}

Este artículo, tiene como objetivo principal analizar la demanda de profesionales de educación superior en el área de humanidades en los municipios de la región 2 valles de Comayagua durante el periodo 2015 - 2016. En la metodología empleada para el desarrollo de esta investigación, se utiliza un enfoque integral; es decir componentes tanto cuantitativos como cualitativos, ya que la información de esta última nos permite profundizar en las causas de las situaciones encontradas en cada municipio y así determinar la pertinencia de las carreras que oferta el CURC.

Dado que se trata de un informe integral, se establecen preguntas de investigación que orientan la búsqueda de información y demás procesos relacionados con la investigación. Tomando como variables la oferta académica, demanda laboral y trayectoria laboral. Además, en la población y muestra se trabaja con los 27 municipios de la región 2 valles de Comayagua, debido que no se conoce una población exacta para hacer un cálculo adecuado. Los instrumentos que se utilizan son la encuesta y la entrevista, las que fueron aplicadas a estudiantes de último año de secundaria, graduados de educación superior, empleadores de profesionales universitarios, emprendedores, líderes comunitarios, ONG, universidades públicas y privadas.

Se recolectaron los datos de los instrumentos aplicados para conocer la pertinencia de estas carreras, se analizaron los resultados y se determinó que, en el CURC, el área de Humanidades, no está ofreciendo carreras de ningún grado académico, y la

\footnotetext{
${ }^{1}$ Asistente Técnico de Laboratorio, Centro Universitario Regional del Centro, Departamento de Agroindustria: sandra.santos@unah.edu.hn

${ }^{2}$ Asesor metodológico, Profesor de la Facultad de Humanidades y Artes, Edwin.medina@unah.edu.hn.
} 
licenciatura de periodismo que se ofrece está ubicada en esta área, pero no pertenece a ella. Igualmente, este Centro Universitario necesita profesionales egresados de la licenciatura en lenguas extranjeras, así como capacitaciones, ya sean cursos, talleres o diplomados.

Palabras clave: Demanda laboral, oferta académica, educación superior, pertinencia, egresados, trayectoria laboral.

\section{ABSTRACT}

In this article research, the main objective is to analyze the demand for higher professional education in the humanities in the municipios of the 2 region of Comayagua valleys in the period 2015 - 2016. In the methodology used for the development of this research, a comprehensive approach is used; meaning both quantitative and qualitative components, since the last information allows us to penetrate in the reasons of the situations found in every municipality and this way to determine the relevancy of the careers that the CURC offers.

Given that it is an integral report, there are established questions of investigation that orientate the search of information and other processes related to the investigation. Taking as variables the academic offer, labor demand and labor path. In addition, at the population and sample one is employed with 27 municipalities of the region 2 Comayagua's valleys, due that no one united nations population to make proper known exact calculation. The instruments that are in use are the survey and the interview, which were applied to students of last year of secondary, graduates of top education, employers of university professionals, entrepreneurs, community leaders, ONG, public and private universities.

The information of the instruments was gathered applied to know the relevancy of these careers, the results were analyzed and one determined that, in the CURC, the area of humanities, it is not offering careers of any academic degree, and the master of journalism that is located in this department does not belong to him. Equally, this university center needs professionals in foreign languages, as well as trainings, already be courses, workshops, graduates or others.

Key Words: Demands of proffessionals, academic offer, higher education, relevance, graduates, career path. 


\section{INTRODUCCIÓN}

En la actualidad una de las preocupaciones más importantes de las instituciones de educación superior es la pertinencia de las carreras que ofertan, por lo que esta investigación sirve al Centro Universitario Regional del Centro como insumo esencial para la toma de decisiones, lo cual facilita el proceso de apertura de nuevas carreras y rediseño de las ya existentes.

Además, Comayagua por ser identificada como ciudad colonial por excelencia, cercanía a la CA-5 y próximamente el Aeropuerto Internacional Palmerola la ciudad se está convertido en un polo de desarrollo para la región, por lo que el CURC debe estar preparado para ofrecer a la sociedad carreras afines al contexto actual y futuro.

Así mismo, esta investigación contribuye al fortalecimiento académico puesto que servirá de insumo para la Universidad Nacional Autónoma de Honduras y en particular al Centro Universitario Regional del Centro para la toma de decisiones en cuanto a apertura de maestrías, diplomados y talleres entre otros. También favorece el desempeño docente en todas las áreas educativas según las necesidades del contexto para que actúe como un formador, capaz de participar en procesos de cambios institucionales y sociales, orientados a perfeccionar la praxis y teoría educativa para una Educación Superior innovadora.

\section{Contenido}

Actualmente, investigadores y expertos apuntan que los universitarios tienen más posibilidades de insertarse cualitativa y cuantitativamente en el mercado laboral, que otras personas con otro tipo de perfil (Arguelles Ma, Quijano García, \& Fajardo, 2014). Sin embargo, la universidad debe readecuar los perfiles profesionales y académicos, los cuales deben estar regulados por una formación basada en competencias y que, a la vez, estos, estén en consonancia con las necesidades de la sociedad. Tomando en cuenta las características generales y cambios del mercado laboral actual y futuro.

En consecuencia, el Centro Regional como institución de Educación Superior debe responder a esos nuevos desafíos que se le presentan, para contribuir al desarrollo económico, político y social de la Región 2 Valles de Comayagua; el cual tiene un gran potencial agrícola, cultural, turístico e histórico. De hecho, la mayor cantidad de museos e iglesias conservadas se encuentran en la restaurada ciudad colonial de 
Comayagua. Dentro de los turistas y visitantes potenciales están los segmentos de mercado internacional; estadounidense, europeo, centroamericano y suramericano y, el mercado nacional; estudiantes y familias. Una vía para lograr este desarrollo es fortaleciendo la formación de recurso humano en el área de humanidades tales como lenguas extranjeras (bilingües y trilingües), ciencias de la educación, arquitectura y otras.

\section{OBJETIVOS}

\section{Objetivo general}

Analizar la demanda de profesionales de educación superior en el área de humanidades en los municipios de la Región 2 Valles de Comayagua, en el periodo 2015 2016.

\section{Objetivos específicos}

1. Caracterizar la oferta académica actual en el área de humanidades en la Región 2 Valles de Comayagua.

2. Determinar la demanda de profesionales de educación superior en el área de humanidades en los municipios de la Región 2 Valles de Comayagua.

3. Establecer la relación entre la oferta académica y demanda de profesionales de educación superior en el área de humanidades.

4. Identificar la trayectoria laboral de los egresados del área de humanidades del CURC.

\section{METODOLOGÍA}

Dado que los objetivos planteados en este estudio pretenden identificar y describir las características de las variables de la investigación, se determinó realizar una investigación descriptiva, ya que lo que se busca es tener información cuantitativa, pero además poder profundizar en las causas de las situaciones encontradas en cada municipio, limitándose a observar y describir el comportamiento de un sujeto sin influir sobre él de ninguna manera. (Hernández Sampieri, Fernández Collado, \& Baptista Lucio, 2010) 
Debido que se trabajó con los 27 municipios de la región 2 valles de Comayagua, no se conoce una población exacta para poder hacer un cálculo adecuado, y en muchos casos se encontró que no existen estudiantes de último año de secundaria, empresas y emprendedores en algunos municipios, por lo que el tamaño de la muestra se realizó mediante un sistema de muestreo no probabilístico de tipo intencional, tratando de tener representatividad de todos los actores involucrados en la investigación. Para efecto de la recolección de información se aplicó 6 tipos de instrumentos; cuestionarios a 182 estudiantes de último año de secundaria, 34 empleadores y 50 graduados universitarios; y entrevistas semi-estructuradas a 2 instituciones de educación superior, 16 líderes comunitarios, y 16 emprendedores. La aplicación de estos instrumentos se realizó en los colegios de educación secundaria públicos y privados, empresas públicas y privadas, universidades públicas y privadas, emprendedores universitarios, alcaldes o vicealcaldes, líderes de patronatos, grupos organizados y egresados de educación superior de cada municipio.

Operacionalización de las variables demanda de profesionales y trayectoria laboral (ver tabla 1):

\section{Tabla 1. Definición conceptual y operacional}

\begin{tabular}{|c|c|c|}
\hline VARIABLE & DEFINICIÓN CONCEPTUAL & DEFINICIÓN OPERACIONAL \\
\hline Demanda de profesionales & $\begin{array}{l}\text { "Son los puestos que demandan las empresas y } \\
\text { que deben ser cubiertos por trabajadores que se } \\
\text { adecuen a los requisitos exigidos por ella. La } \\
\text { demanda la constituyen la totalidad de puestos de } \\
\text { trabajo vacantes, que son ofrecidos por las } \\
\text { empresas a los trabajadores". (Fontana, 2003; 122) }\end{array}$ & $\begin{array}{l}\text { Esta variable se operacionalizará a través de } \\
\text { cuestionarios aplicados a empleadores y } \\
\text { estudiantes de último año de secundaria. }\end{array}$ \\
\hline Tray ectoria laboral & $\begin{array}{l}\text { Según Johnny Javier Orejuela Gómez y Andrés } \\
\text { Correa García (2007), Trayectoria laboral es el } \\
\text { recorrido o transito particular de las experiencias de } \\
\text { un individuo en el campo laboral, que ilustran los } \\
\text { desplazamientos bajo ciertas coordenadas } \\
\text { determinadas de manera simple y que describen } \\
\text { posiciones especiales ocupadas en ese campo } \\
\text { relacional, respecto de un punto de origen, que es } \\
\text { el momento de inserción, y de un punto final, que } \\
\text { es la desmercantilización. }\end{array}$ & $\begin{array}{l}\text { Esta variable se operacionalizará a través de } \\
\text { cuestionarios aplicados a graduados } \\
\text { universitarios del área de humanidades. }\end{array}$ \\
\hline
\end{tabular}

Fuente: Elaboración propia.

Matriz de categorización (ver tabla 2): 


\section{Tabla 2. Categorías de análisis y definición}

\begin{tabular}{|c|l|}
\hline $\begin{array}{c}\text { CATEGORIAS DE } \\
\text { ANÁLISIS }\end{array}$ & \multicolumn{1}{c|}{ DEFINICIÓN CONCEPTUAL } \\
\hline Oferta académica & $\begin{array}{l}\text { Según Adalberto Gabriel Díaz Torres (2008), señala que oferta académica flexible es la } \\
\text { alternativa estratégica operativa en la educación superior, para hacer de la enseñanza y el } \\
\text { aprendizaje del conocimiento un servicio de calidad para la satisfacción del estudiante, el } \\
\text { mejor desempeño docente y el mejor servicio de la administración, ligando la actividad } \\
\text { académica al desarrollo del hombre y la sociedad, movilizando las estructuras de } \\
\text { enseñanza, aprendizaje e investigación en beneficio de toda la comunidad, ajustándose } \\
\text { continuamente a las exigencias de la demanda diversificada de la población estudiantil. }\end{array}$ \\
\hline Demanda de profesionales & $\begin{array}{l}\text { "Son los puestos que demandan las empresas y que deben ser cubiertos por trabajadores } \\
\text { que se adecuen a los requisitos exigidos por ella. La demanda la constituyen la totalidad de } \\
\text { puestos de trabajo vacantes, que son ofrecidos por las empresas a los trabajadores". } \\
\text { (Fontana, 2003; 122) }\end{array}$ \\
\hline
\end{tabular}

Fuente: Elaboración propia.

En síntesis, la metodología aplicada para la realización de esta investigación hizo acopio de los siguientes pasos:

a. Determinar la región donde se llevará a cabo la investigación. (Ver mapa 1)

\section{Mapa 1. Ubicación de la región 2 valles de Comayagua}

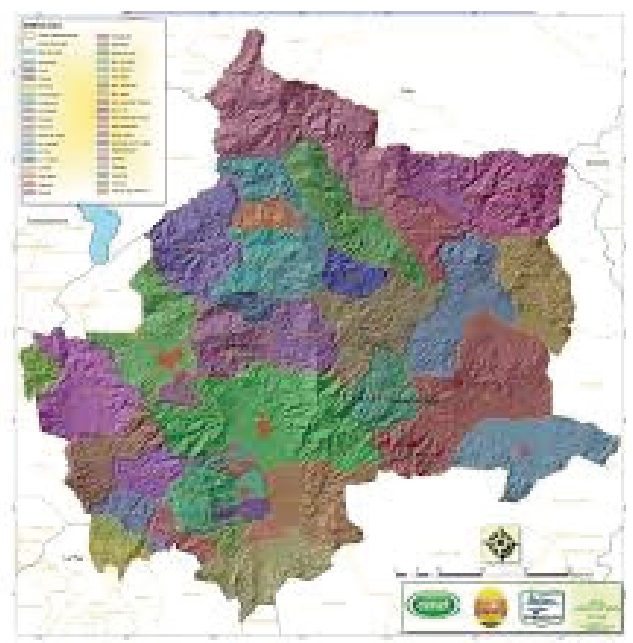

Fuente: PDR-OT 
Esta región comprende 37 municipios de 5 departamentos; 19 que corresponden a Comayagua, 6 a Francisco Morazán, 3 a Intibucá, 7 a La Paz y 2 a Yoro. En esta investigación se trabajaron solo con 25 de ellos. Para la selección se tomaron en cuenta características como: ubicación o acceso del CURC, estatus socioeconómico y mayor afluencia de estudiantes en el CURC según su origen. Cabe mencionar que, aunque los municipios de Marcala y La Esperanza no pertenecen a esta región, se consideraron para este estudio porque en el CURC existe matrícula de estudiantes de los municipios antes mencionados.

b. Se aplicaron los instrumentos a los diferentes actores de cada municipio. En vista que algunos municipios aún no tienen estudiantes de último año de secundaria, estos se completaron con estudiantes de otros centros educativos de municipios que cuentan con varios colegios públicos y privados.

c. Se recopilaron los datos de los cuestionarios aplicados a estudiantes de último año de secundaria, empleadores y graduados universitarios y; las entrevistas aplicadas a instituciones de Educación Superior, líderes comunitarios y emprendedores.

d. Finalmente, se ingresaron los datos en la base de datos, se tabularon y se elaboraron los gráficos en SPSS y Microsoft Excel de las variables demanda de profesionales y trayectoria laboral. Así mismo, se realizaron las transcripciones de las entrevistas aplicadas para hacer su respectivo análisis.

\section{RESULTADOS Y DISCUSIÓN}

\section{a) Oferta académica}

Al hacer una revisión de la oferta académica de las diez universidades ubicadas en la región 2 valles de Comayagua, se observa claramente que en el área de las humanidades la Universidad Pedagógica Nacional Francisco Morazán, es la que tiene mayor oferta. La Universidad Católica, UCRISH, la Metropolitana, la UTH y la Universidad de Defensa no ofrecen ninguna carrera del área en mención (Ver tabla 3).

En el Centro Universitario Regional del Centro, la única carrera que oferta y que está ubicada en el área de humanidades, es la Licenciatura en Periodismo. Es oportuno señalar que esta carrera no es del área de humanidad sino de sociales, puesto que según la Facultad de Humanidades y Artes de la UNAH; las carreas que incluye son Música, Arquitectura, Filosofía, Educación Física, Técnico interprete Lengua de Señas, Lenguas Extranjeras, Pedagogía y Letras, y con grado de maestría; Actividad 
Física para la Salud, Literatura Centro Americana, Docencia Superior y Tecnología Educativa.

Tabla 3. Oferta académica

\begin{tabular}{|l|l|}
\hline UNIVERSIDADES & \multicolumn{1}{|c|}{ OFERTA ACADÉMICA } \\
\hline \multirow{2}{*}{ 1. UJCV } & a. Turismo \\
\cline { 2 - 2 } & b. Arquitectura \\
\hline 2. CRAED & a. Pedagogía y ciencia de la educación. \\
\hline 3. POLITECNICA & a. Gerencia en turismo \\
\hline 4. CURC & a. Periodismo \\
\hline 5. UPNFM & a. Letras \\
\cline { 2 - 2 } & b. Educación básica \\
\cline { 2 - 2 } & c. Inglés \\
\cline { 2 - 2 } & d. Francés \\
\cline { 2 - 2 } & e. Formación pedagógica \\
\cline { 2 - 2 } & f. Competencias docentes \\
\hline
\end{tabular}

Fuente: Elaboración propia.

Con esta investigación el CURC, puede ofertar nuevas carreras y del área de Humanidades, incorporando como eje transversal el emprendimiento para estar en correspondencia con los nuevos desafíos de la región 2 valles de Comayagua.

b)Demanda de profesionales

\section{Líderes}

De acuerdo con la información obtenida de las entrevistas realizadas a los diferentes líderes de los municipios de la región 2 valles de Comayagua, municipios como La Paz, Comayagua, La Villa de San Antonio y Jesús de Otoro, mencionaron que uno de los desafíos que prevén para el desarrollo económico es el turismo en sus distintas ramas como el turismo cultural, religioso, gastronómico, de aventura y el ecoturismo. Y aunque el turismo en si no es parte del área de Humanidades, podemos rescatar el hecho de que los municipios arriba mencionados a excepción de Jesús de Otoro, se les prevé un crecimiento económico latente por su ubicación. Situación que se debe aprovechar para ofertar carreras del área de Humanidades como lo es lenguas extranjeras para atender los turistas que en la actualidad no son atendidos de la forma apropiada por no contar con profesionales del área. 
Al consultársele a los líderes sobre el grado académico que requieren para atender estas necesidades, un $33.3 \%$ respondió que, con grado de licenciatura, un $33.3 \%$ grado de técnico y un $33.3 \%$ con especialidades. Esto marca las pautas para crear carreras en los diferentes grados académicos y así, poder atender la demanda futura de profesionales en el área de Humanidades: Lenguas Extranjeras.

\section{Emprendedores}

Al hacer un análisis sobre la información obtenida a través de entrevistas aplicadas a los emprendedores del área de humanidades, el 50\% corresponde a hombres y el $50 \%$ a mujeres. Esto nos indica que ambos sexos tienen la capacidad de emprender sus propios negocios sin discriminación de sexo, aunque en investigaciones realizadas afirman que existe mayor temor al fracaso en las mujeres, en nuestro contexto social podemos afirmar lo contrario. En el área de humanidades el emprendimiento es una labor realizadas de forma equitativa para ambos sexos.

Podemos afirmar que el mayor porcentaje de emprendedores (33.3\%) lo representan los graduados de Lenguas Extranjeras, los que obtuvieron licenciatura en Pedagogía, Periodismo, Educación Básica y en Turismo tienen poca participación en este tema. Para este estudio, se entrevistaron a graduados de las distintas universidades de la región 2 valles de Comayagua, y si bien es cierto que hay emprendedores en todas las áreas del conocimiento, estos son muy pocos en comparación con la mayoría de los emprendedores que ni siquiera han culminado sus estudios de primaria y secundaria.

Este estudio nos permitió descubrir que el 83\% de los emprendedores de la región 2 valles de Comayagua, están en el municipio de Comayagua y Jesús de Otoro. Esto quizás porque de todos los municipios visitados, Comayagua es el que tiene mayor desarrollo socioeconómico, y seguirá creciendo aún más con la construcción del aeropuerto internacional, pues esto traerá como consecuencia el incremento de turistas. Además, es oportuno señalar que Jesús de Otoro es uno de los municipios que forma parte de la ruta lenca, la cual es considerada como un potencial turístico. Después de lo anterior expuesto, podemos aseverar que hay una demanda de profesionales latentes de carreras como lenguas extranjeras y turismo.

Algunas de las empresas creadas por los emprendedores universitarios tenemos: escuelas de enseñanza del idioma inglés, canales de televisión, diagramación y otras. 
Según lo expresado por los emprendedores, de las únicas instituciones que han recibido apoyo técnico son de proveedores y CENET, del Centro Universitario Regional del Centro no han recibido apoyo técnico. Se observa claramente la oportunidad para diseñar cursos, talleres, seminarios o diplomados en temas afines al área de estudio para apoyar a través de capacitaciones a los graduados-emprendedores del CURC y otros.

\section{Empleadores}

Existe una diferencia notoria entre el grado académico obtenido por los empleados universitarios del área de humanidades y las necesidades expresados por los empleadores de la sociedad. Se puede observar que hay demanda de profesionales con grado de maestría y especialidad (Ver gráfico 1).

Gráfico 1. Grado académico de los empleados versus la requerida por los empleadores

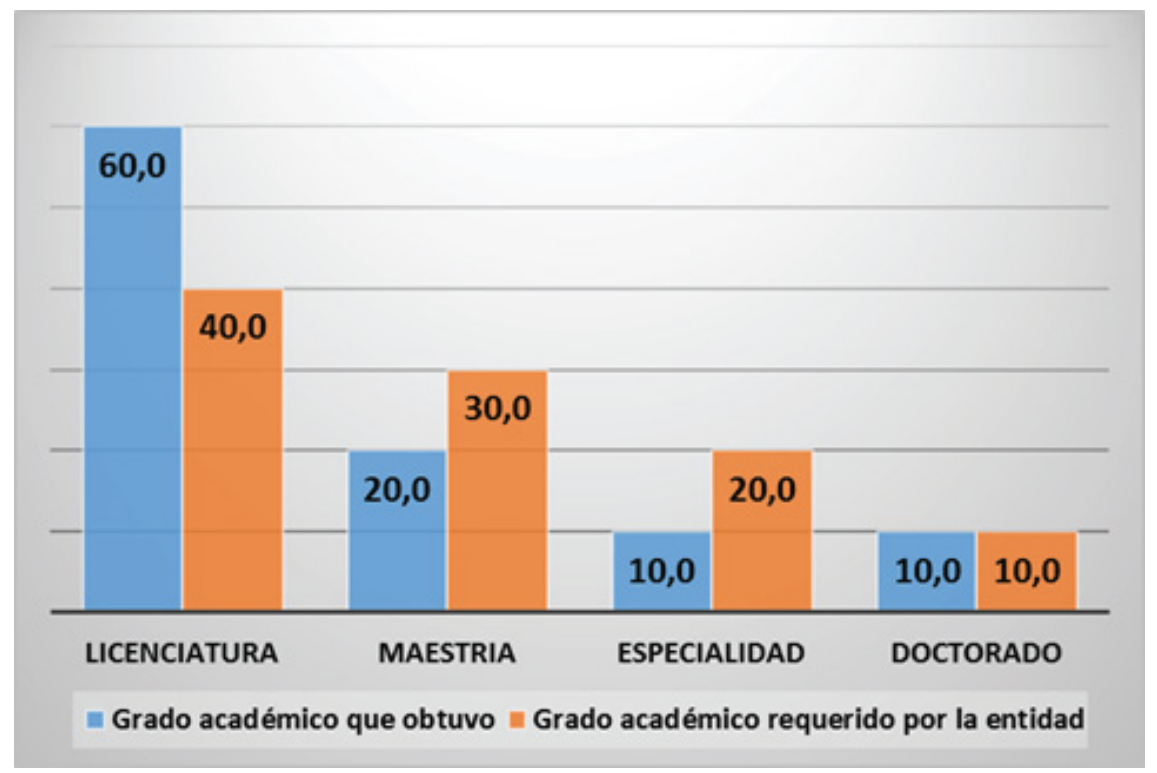

Fuente: Elaboración propia.

Se puede considerar que en los profesionales del área de Humanidades existe una fuerte debilidad en el uso de la tecnología y en las actitudinales, por lo que la UNAH-CURC debe incorporarlas en el diseño curricular de las carreas como ejes transversales para satisfacer las necesidades expresadas por los empleadores (Ver gráfico 2). 
Gráfico 2. Competencias ausentes en los profesionales del área de humanidades.

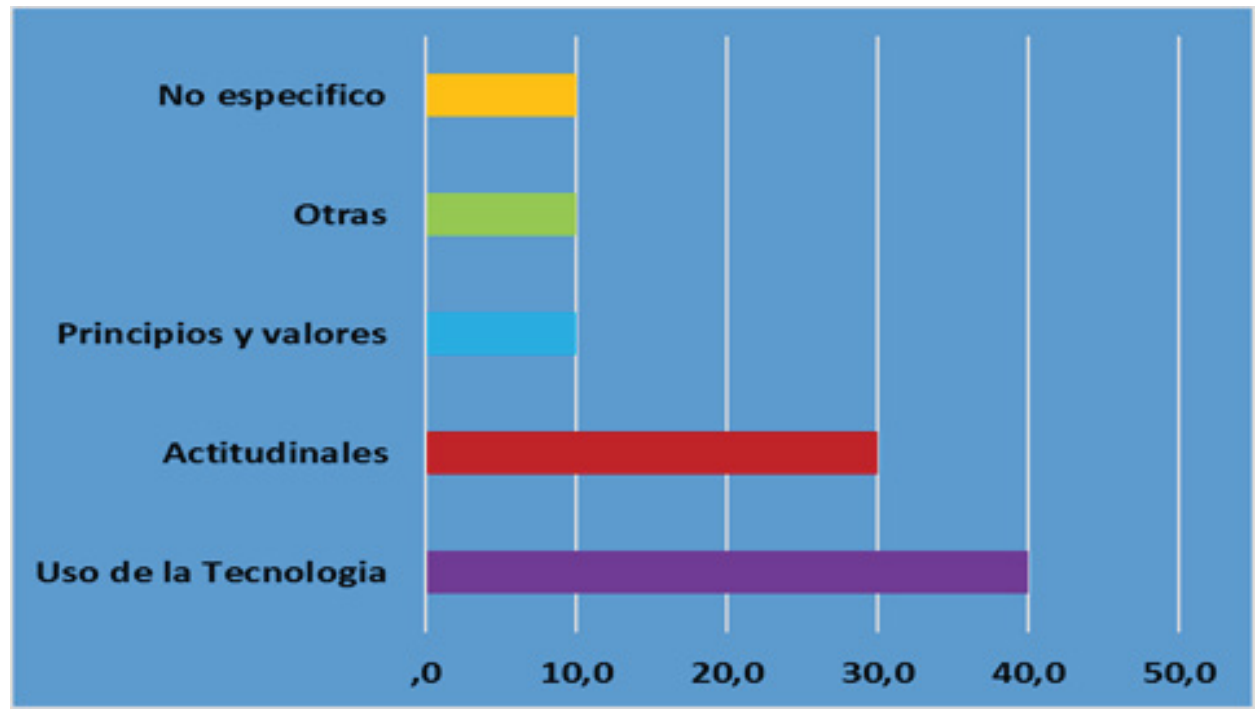

Fuente: Elaboración propia.

Estudiantes de último año de secundaria

Se puede ver que existe un alto interés en proseguir estudios universitarios, sin embargo, las situaciones socioeconómicas (ingreso mensual de los padres muy bajo, familias numerosas, padres que solo han cursado la primaria, etc) de la mayoría de los municipios limitan en muchos casos las posibilidades (Ver gráfico 3).

Gráfico 3. Interés de los estudiantes de último año de secundaria en seguir estudios universitarios

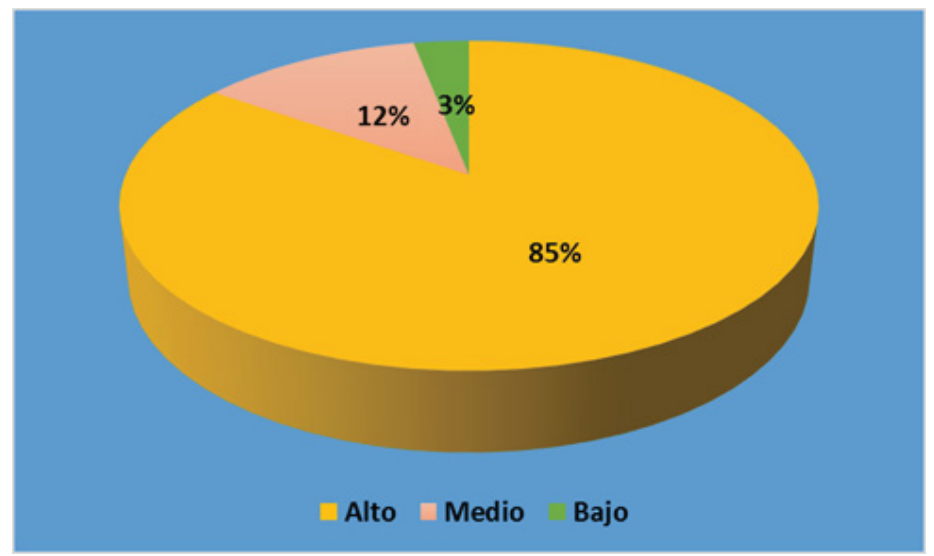

Fuente: Elaboración propia. 
La necesidad mostrada por los estudiantes de último año de secundaria en estudiar la licenciatura en lenguas extranjeras es muy alta. Tomando en cuenta que, por sus características, Comayagua se ha convertido en un polo de desarrollo, es oportuno para esta institución dar respuesta a esta demanda de profesionales (Ver gráfico 4).

\section{Gráfico 4. Carrera que le gustaría estudiar}

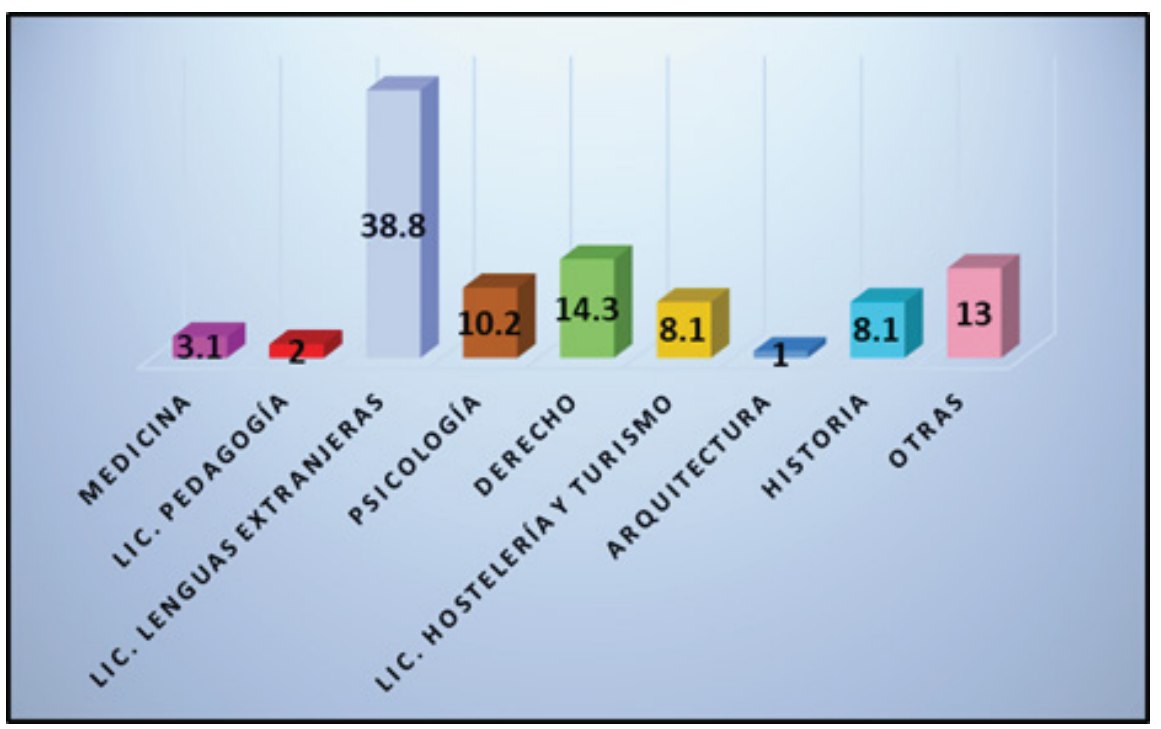

Fuente: Elaboración propia.

c) Relación entre oferta académica y demanda de profesionales

Al hacer el análisis de la relación entre oferta académica y la demanda de profesionales en Educación Superior en el área de humanidades de la región 2 valles de Comayagua, y según los resultados obtenidos, el Centro Universitario Regional del Centro no está ofreciendo carreras que estén en correspondencia con la demanda laboral.

Comayagua está creciendo de forma acelerada y sumada a ella están algunos municipios como la Villa de San Antonio, La Paz, Jesús de Otoro y Marcala. Según los empleadores, líderes comunitarios, emprendedores, estos municipios necesitan de profesionales con grados de licenciatura y técnicos en el área de humanidades, así como talleres, cursos, diplomados y algunas especialidades.

Carreras como lenguas extranjeras es muy importante, ya que el crecimiento de turistas se ha previsto que incrementara una vez construido el Aeropuerto Internacional de Palmerola. 
d) Trayectoria laboral de egresados

Aunque la mayoría de los graduados del área de humanidades están laborando, es importante que el Centro Universitario Regional del Centro de seguimiento a sus egresados, en este caso, a los profesionales del área de Humanidades, para conocer sus necesidades académicas (Ver gráfico 5).

Gráfico 5. Graduados del área de humanidades que están laborando.

Fuente: Elaboración propia.

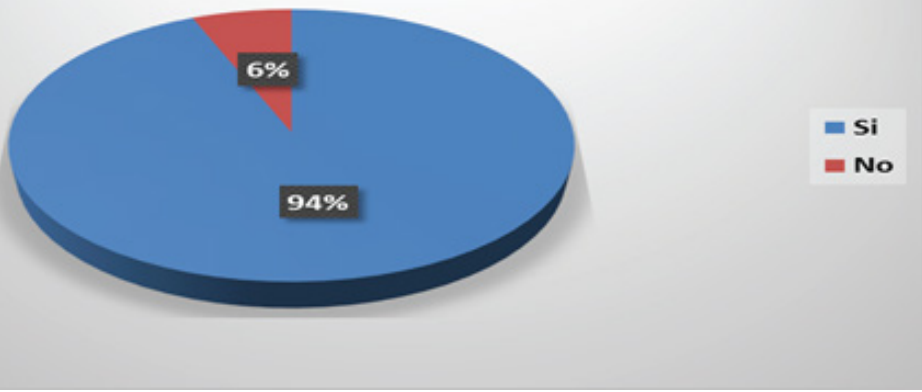

Es de resaltar que, aunque la mayoría de graduados del área de humanidades se desempeñan en instituciones donde realizan actividades afines a su perfil, también hay un buen porcentaje de profesionales que no se desempeñan en el área de su profesión. Siendo esta una oportunidad para que las autoridades pertinentes evalúen el plan de estudio de la carrera de periodismo y hacer un rediseño de la misma (Ver gráfico 6).

Gráfico 6. Los graduados se desempeñan en el campo de su profesión.

Fuente: Elaboración propia.

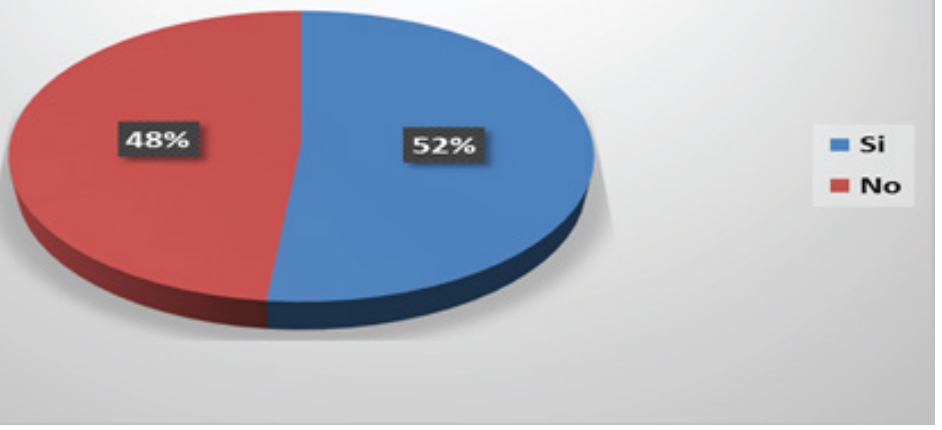




\section{CONCLUSIONES}

1. El centro Universitario Regional del Centro en el área de Humanidades no está ofreciendo carreras de ningún grado académico y la licenciatura de periodismo que está en humanidades no pertenece a esta área.

2. En cuanto a la demanda de profesionales de educación superior en el área de Humanidades en los municipios de la Región 2 Valles de Comayagua, está necesitando de profesionales de lenguas extranjeras en los diferentes grados académicos.

3. Entre la oferta académica y demanda de profesionales de educación superior en el área de Humanidades, no existe correspondencia, pues no está ofreciendo las carreras que actualmente está demandando esta región.

4. Puesto que, en el CURC la Licenciatura de periodismo está en el área de Humanidades, al investigar sobre la trayectoria laboral de sus egresados, un buen porcentaje mostró interés en recibir formación académica, ya sean cursos o diplomados del tipo presencial ofertados por el CURC, pues, la mayoría de las capacitaciones recibidas son de otras instituciones. Cabe mencionar que, entre los profesionales de la comunicación, el emprendimiento se manifiesta tanto en hombres como mujeres en un mismo porcentaje. Sin embargo, son pocos los profesionales emprendedores.

\section{AGRADECIMIENTO}

Quiero agradecer a la Dirección de Investigación Científica y Posgrado de la UNAH, por su apoyo financiero y técnico en la elaboración del presente artículo científico. Este agradecimiento se extiende al Dr. Edwin Medina, por sus conocimientos, sus orientaciones, su paciencia y su motivación que han sido fundamentales durante el proceso formativo como investigador. De igual manera a todas las instituciones y personas que han colaborado en la presente investigación.

\section{REFERENCIAS BIBLIOGRÁFICAS}

Balmes, J. (2014). Encuesta de competencias profesionales 2014. Obtenido de http://www.uv.mx/bolsadetrabajo/files/2014/10/encuesta_competencias_profesionales 
Brunner, J. J. (Mayo de 2005). Tendencias recientes de la educación superior a nivel internacional: Marco para la discusión sobre procesos de aseguramiento de la calidad. Obtenido de file:///C:/Users/Sandrita/Downloads/Articulo\%20Brunner.pdf

C. Vessuri, H. M. (Mayo-Agosto de 1993). Desafíos de la edducación superior en relacción con la formación y la investigación ante los procesos económicos actuales y los nuevos desarrollos tecnológicos. Obtenido de http://rieoei.org/oeivirt/rie02a06.htm

Carot, J. M. (Mayo de 2011). La universidad y el campo laboral. Obtenido de http://www.iesalc.unesco.org.ve/index.php?option=com_content\&view=article\&id=2691:entrevistaa-jose-miguel-carot-a-los-egresados-cada-vez-mas-se-les-demanda-flexibilidad-ycapacidad-de-adaptacion\&catid $=32 \&$ ltemid $=600 \&$ lang $=e s$

Diaz Torres, A. G. (S/A). La flexibilización de la oferta académica mejora la calidad del aprendizaje. Recuperado el 18 de Septiembre de 2015, de http://www.rieoei.org/deloslectores/1332Diaz.pdf

Guzmán, J. (Enero de 2011). La calidad de la enseñanza en la educación superior. ¿Qué es una buena enseñanza en este nivel educativo? Obtenido de http://www.scielo.org.mx/scielo.php?script=sci_arttext\&pid=S0185-26982011000500012

Huneeus, C. (1972). Concepto y fundamentos de la reforma universitaria en América Latina. Obtenido de http://www.analesderecho.uchile.cl/index.php/ACJYS/article/view/4315/42 Inserción profesional de los egresados de la Licenciatura en Administración Turística. (S/F). Obtenido de http://insercionlaboralegresadoss.jimdo.com/marco-te\%C3\%B3rico/definici $\%$ C3\%B3n-de-conceptos-b\%C3\%A1 sicos/

La Tribuna. (21 de Diciembre de 2015). Aeropuerto internacional Palmerola estará listo en el 2018. Obtenido de http://www.latribuna.hn/2015/12/21/aeropuerto-internacional -palmerola-estaria-listo-en-el-2018/

López Segrera, F. (Julio de 2008). Tendencias de la educación superior en el mundo y en América Latina y el Caribe. Obtenido de http://www.ses.unam.mx/curso2014/pdf/LopezSegrera2008.pdf

Orejuela Gomez, J. J. (2007). Trayectorias laborales y relacionales. Recuperado el 26 de Septiembre de 2015, de http://www.redalyc.org/pdf/1053/105316864005.pdf

Sánchez D., I. (Octubre de 2011). Los desafíos de la educación superior en Chile. Obtenido de file:///C:/Users/Sandrita/Downloads/Desafios_de_la_Educacion_Superior.pdf

Secretaría de trabajo y seguridad social. Observatorio del mercado laboral. (Mayo de 2011). Aantecedentes y diagnóstico del mercado laboral. Obtenido de http://www.trabajo.gob.hn/organizacion/dgt-1/direccion-general-de-empleo/oml/ANTECEDENTES\%20Y \%20DIAGNOSTICO\%20DEL\%20MERCADO\%20LABORAL-1.pdf

UNAH-CURC, CENET, \& ESNACIFOR. (2013). Plan de Desarrollo Regional con Enfoque de Ordenamiento Territorial (PDR-OT), Región 2, Valles de Comayagua. Comayagua. 